\title{
Disgerminoma puro de ovario Presentación de dos casos
}

\author{
Guillermo Acosta Osio MD*; María Cristina Marceles; Jaime Orozco; Alfredo Barraza**
}

\begin{abstract}
RESUMEN: Siendo el Disgerminoma el tumor de células germinales más común en mujeres jóvenes, es también de los menos frecuentes de los tumores de ovario, oscilando su incidencia en Colombia del 0.48 a $0.55 \%$.

Se presentan dos casos atendidos en el Departamento de Ginecología y Obstetricia del Hospital Universitario Metropolitano en un período de 10 años.
\end{abstract}

PALABRAS CLAVES: Disgerminoma, tumores de ovario, presentación de dos casos.

SUMMARY: Being the Disgerminoma the tumor of germinal cells most common among young women, is also one not so frecuent of ovarian tumors, oscilating its incidence in Colombia between 0.48 and $0.55 \%$.

There has been two cases presented in the department of Ginecology and Obstetrics of the Metropolitana University Hospital during a period of ten $(10)$ years.

KEY WORDS: Disgerminoma, ovarian tumors, presentation of two cases.

El Disgerminoma es el tumor de células germinales más común y ocurre principalmente en mujeres jóvenes; aproximadamente $40-45 \%$ se encuentra en mujeres menores de 20 años de edad y $80 \%$ antes de los 30 años (1-3).

Es también el más común de todos los tumores malignos que ocurren en estados intersexuales en los que hay una gónada disgenética; estas neoplasias pueden ser asintomáticas y cerca del $15 \%$ se presenta durante el embarazo como causa de la distocia o durante la cesárea, junto con el cistadenoma seroso es el tumor ovárico más común en el embarazo $(2,4$ 5). Quizás es el tumor sólido más radiosensible que se conoce; tiene predilección por propagarse linfáticamente (1$2,6)$.

La frecuencia del disgerminoma es aproximadamente del 1 al 2\% de todas las neoplasias ováricas malignas (1-3).

Este es un tumor redondeado de tamaño muy variable, rosado-amarillento con algún punteado hemorrágico, se caracteriza por presentar masas celulares con patrón insular y a veces travecular. Las células son redondeadas, ovaladas o poligonales, con citoplasma abundante y pálido, con núcleo grande. El estroma es fibroso y casi siempre infiltrando los linfocitos (1-2).

Los disgerminomas son hormonalmente inactivos, pero pueden producir pequeñas cantidades de alfafetoproteína o

* Profesor y Director Dpto. Ginecología y Obstetricia. Universidad Metropolitana. Hospital Universitario Metropolitano.

** Residentes de II Año Ginecología y Obstetricia. Universidad Metropolitana. Hospital Universitario Metropolitano. de gonadotropina coriónica humana y son de una variedad mixta (6).

A continuación citaremos dos casos considerándolos interesantes dada la escasa frecuencia con que se presenta esta patología:

\section{Caso número 1}

Mujer de 20 años GO PO AO originaria de Baranoa (Atlántico) HC No. 10231 quien consultó al Hospital Universitario Metropolitano por presentar una masa supraclavicular izquierda de más o menos $8 \mathrm{cms}$, acompañada de dolor torácico y fiebre no cuantificada. FGO: Menarquia: 13 años, ciclos 30x4, FUM: 19-XII-89. Amenorrea de 8 meses. Inicio Vida Marital: Negativo. Dismenorrea: positiva. Citología: nunca.

Flujos vaginales: negativo. Se le encontró pálida, no ictérica, afebril, delgada, con una masa en mesogastrio, flanco y fosa ilíaca izquierda, sólida y lobulada que ocupaba hemiabdómen inferior, de $20 \mathrm{cms}$, móvil, no dolorosa.

Previo a su ingreso a hospital, acudió a consultorio privado donde se le realizó ecografía que reportaba masa sólida y lobulada de $18.5 \mathrm{cms}$ x $9.7 \mathrm{cms} \times 12.3 \mathrm{cms}$ en su diámetro longitudinal, anteroposterior y transversal respectivamente en útero, correspondiente a miomatosis. Urografía excretora dentro de lo normal; colon por enema con zona de compresión a nivel del sigmoide por desplazamiento de la masa, resto de colon dentro de los límites normales. Al tacto rectal se palpó masa dura que parecía corresponder a útero miomatoso, por lo cual se programa para miomectomía e 
histeroplastia, pero con biopsia transoperatoria de masa supraclavicular y sin descartar la posibilidad de tumoración ovárica debido a su edad. Al realizar la laparotomía, se encontró tumor de ovario izquierdo de aproximadamente 22 $\mathrm{cms}$, lobulado, adherido completamente a útero, de naturaleza maligna, compatible con un disgerminoma de ovario izquierdo con metástasis a trompa y ovario derecho. Se realizó histerectomía, apendicectomía, omentectomía y salpingooforectomía bilateral, además de extirpación de ganglios preescalénicos hipertrofiados.

En el estudio histopatológico No. BP 526 se informó:

«Disgerminoma puro de ovario izquierdo con componente linfoide y reacción granulomatosa con compromiso tumoral metastásico a trompa y ovario derecho, líquido peritoneal positivo para células malignas, ganglios preescalénicos con tumor maligno» El Comité Oncológico ordenó quimioterapia la cual no se llevó a cabo por carecer la paciente de recursos económicos, por lo cual se le ordenó radioterapia con 43 sesiones en total, se le solicitó antígeno carcinoembrionario, CA 125 y perfil de la función hepática y renal con pruebas de coagulación, valoración por neumología, todo lo anterior dentro de límites normales, posterior a la radioterapia. Se revisa por última vez el 6 de agosto de 1990 y se le pide control radiográfico pulmonar y ecografía ginecológica, pero la paciente no regresa más, enterándonos que falleció a los 6 meses sin ser posible determinar más datos.

\section{Caso número 2}

Paciente femenina de 24 años de edad natural de Viola (Cundinamarca) HC No. 94996 procedente y residente en Barranquilla, quien consultó por masa en hipogastrio al Hospital Universitario Metropolitano, la cual se le empezó a notar hacia un año, no dolorosa y sin sintomatología, la cual tuvo un crecimiento rápido, por lo cual le ordenaron ecografía que reportó masa en ovario derecho. Ficha Gineco-Obstétrica: Menarquia: 10 años, Ciclos: 28x3. Inicio Vida Marital: Negativa. GO PO AO. Al examen físico se encontró abdomen blando, depresible, no doloroso, se palpó masa de más o menos unos $20 \mathrm{cms}$, móvil, en hipogastrio y fosa ilíaca derecha.

Se le realizó laparotomía el día 19 de septiembre de 1988 encontrándose masa de más o menos $25 \times 20 \mathrm{cms}$ en ovario derecho, sólido, de color grisáceo de 3.000 grs. de peso y un ovario izquierdo poliquístico; se realizó ooforectomía derecha. Diagnóstico transoperatorio: Disgerminoma. Ovario contralateral con síndrome de ovario poliquístico y sin tumor.

En su post-operatorio evolucionó satisfactoriamente; continúa en el comité oncológico.

Se recibió informe de patología que reportó «Disgerminoma de escaso contenido linfoide e invasión capsular» (No. BP 485).
Luego se le realizó seguimiento por la consulta externa con marcadores tumorales, ecografía, alfafetoproteína, HCG, evolucionando hasta la fecha satisfactoriamente.

\section{Comentarios}

El Disgerminoma constituye del 1 al $2 \%$ de los tumores de ovario y ocurre con mayor frecuencia en niñas y mujeres jóvenes (1-2). En Colombia parece ser más raro, según el estudio de Guzmán va de 0.48 a $0.55 \%$ en relación con todas las neoplasias ováricas (7).

Estos dos casos han sido los únicos en 10 años que se han presentado en el Hospital Universitario Metropolitano. Como podemos observar en los casos citados, son dos pacientes jóvenes de 20 y 24 años respectivamente, una con metástasis y la otra con invasión a cápsula pero sin extensión, en las cuales las conductas tomadas son diferentes debido al estadío que mostraba cada una de las pacientes. También podemos observar que una de las manifestaciones iniciales es la presencia de la masa en hipogastrio y casi siempre no produce ningún efecto característico sobre la menstruación, aunque el disgerminoma se presenta a menudo en mujeres que han padecido amenorrea a consecuencia de una deficiencia gonadal.

El motivo de consulta de nuestras pacientes y el desarrollo de la patología es semejante a los diferentes artículos publicados, (7-10) en los cuales incluyen revisión de grandes series.

En cuanto al tratamiento, como la mayor parte de estas pacientes son jóvenes, existe la tendencia a evitar la cirugía radical -siempre que sea posible- tratándose de pacientes jóvenes que presenten tumores bien encapsulados y unilaterales como en el caso 2 , en la cual está plenamente justificada la cirugía conservadora. Se recomienda cirugía conservadora practicando salpingo-ooforectomía, con radioterapia o no de los ganglios pre-aórticos y con protección de la otra gónada.

Las recidivas pueden tratarse con radioterapia ya que es conocida la gran radiosensibilidad de este tumor, y es así como Björkholm y cols. (10), consideran la radioterapia como un efectivo tratamiento complementario postcirugía en su revisión de 60 pacientes.

Es importante tener en cuenta el estadío pues como publicó Gallion (9) en el estadío I la supervivencia a 5 años llega al 91\%, mientras que en el estadío IIa al IV, es el $65 \%$ y la supervivencia de estas pacientes tratadas con quimioterapia es comparable a las tratadas con radiación, siendo considerada la quimioterapia con etoposido, bleomicina y cisplatino por Gershenson (11) como una alternativa a la radioterapia en el tratamiento del disgerminoma metastásico.

\section{Agradecimientos} crito.

A la señora Leomy Durán por la transcripción del manus- 
Figura 1

TUMOR A PUNTO DE SER EXTRAIDO DE LA CAVIDAD ABDOMINAL



Figura 3

ASPECTO DEL FONDO DE SACO POST HISTERECTOMIA



Figura 5

DETALLE DE UTERO Y ANEXOS CONTRA LATERALES METASTASICOS



Figura 2

SE TIENE PINZADO ANEXOS. SE OBSERVA LA ADHERENCIA DEL DISGERMINOMA YA LIBERADA A FONDO UTERINO Y EPIPLON

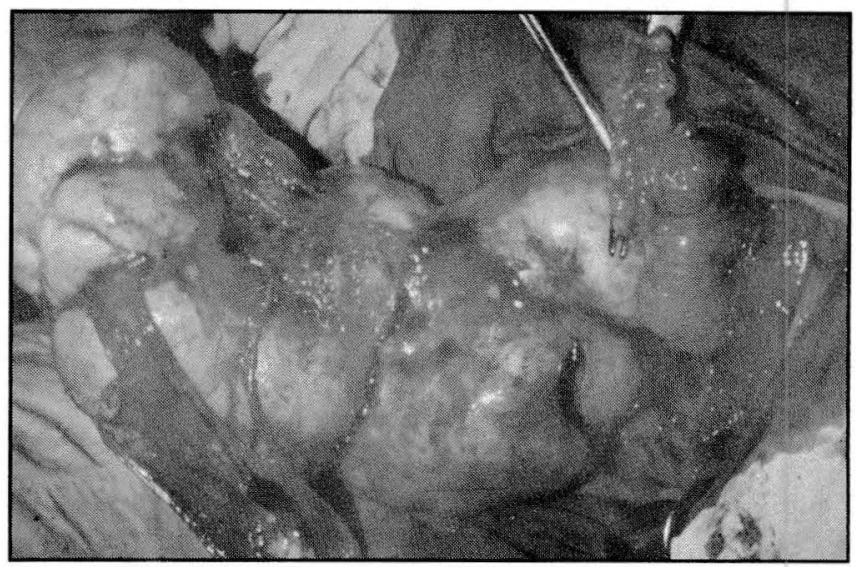

Figura 4

PIEZA QUIRURGICA COMPLETA

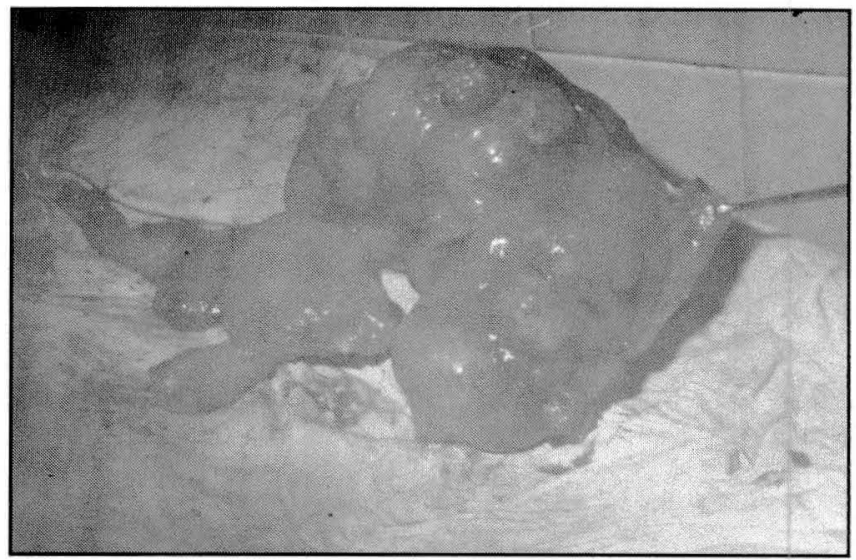

Figura 6

DETALLE CORTE DE PATOLOGIA MACROSCOPICA

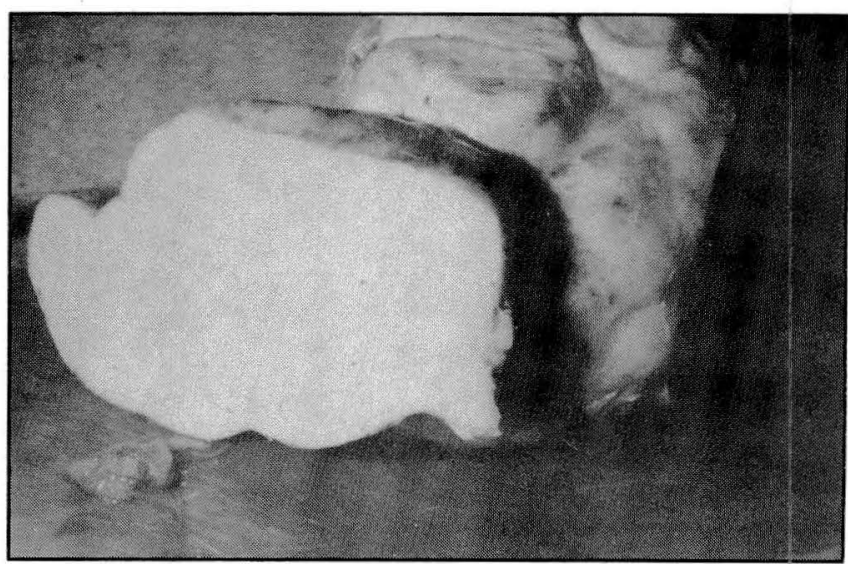




\section{BIBLIOGRAFIA}

1. González-Merlo y cols. Oncología ginecológica. Editorial Salvat. Mallorca, Barcelona. 1991; 349-352.

2. DiSaia Philips., Creasman W. Ginecología Oncológica. Editorial Panamericana. Buenos Aires, Argentina. 3a. edición. 1991; 306-310.

3. Benson S. Diagnóstico y tratamiento en Gineco-Obstetricia. Editorial El Manual Moderno. Buenos Aires, Argentina. 3a. edición. 1983; 325-326.

4. Botero J., Jubiz A., Henao G. Obstetricia y Ginecología. Medellín, Colombia, 4a. edición. 1990; 600.

5. Ridley J., Te Linde R. Cirugía Ginecológica. Editorial Interamericana. 1978; 784-785.

6. Gallup DG., Tallado E. Clínicas Obstétricas y Ginecológicas. 1987; 3: 633-634.
7. Guzmán RD., Fonegra A., Alvarado R., Moreno A. Disgerminoma. Rev. Col. Obstet. Ginecol. 1976; 27(6): 313-322.

8. Akhtar M., Bakri Y., Rank F. Dysgerminoma of the Ovary with Rhabdomyosarcoma Cancer. 1989; 64: 2309-2312.

9. Gallion H., Van NJ., Donaldson E. and Powell D. Ovarian Dysgerminoma: Report of seven casos and review of the literature. Am. J. Obstet. Gynecol. 1988; 158: 591.

10. Björkholm E., Lundell M., Gyftodimos A. and Silfverswärd C. Dysgerminoma. Cancer. 1990; 65: 38-44.

11. Gershenson D., Wharton T., Kline $R$ et al. Remisión completa quimioterapéutica en pacientes con disgerminoma ováricos metastásicos; potencial para la cura y preservación de la capacidad de reproducción. Cáncer. 1986; 58: 2594-2599. 